\title{
CORPORATE ENTREPRENEURSHIP: A LITERATURE REVIEW AND FUTURE RESEARCH PERSPECTIVES
}

\author{
Magdalena Popowska* iD https://orgid.org/0000-0002-2235-7196
}

\begin{abstract}
Background. Nowadays, corporate entrepreneurship (CE) is perceived as an essential approach to boost the innovation and creativity within existing organizations for achieving higher opportunities in the market. This paper examines this concept, which has been largely discussed in the Anglo-Saxon world over the last thirty years. Like for many other phenomena, also in case of $\mathrm{CE}$, this discussion has provided numerous conceptualizations, and consequently, there is not one unique definition of $\mathrm{CE}$. In this respect, the search for an appropriate basis for understanding and describing the phenomenon of CE engenders a challenging issue for entrepreneurship researchers.
\end{abstract}

Research aims. This paper aims at creating a large platform for understanding the concept of CE by means of a clarification effort through the review of the most important papers in this field and identification of the existing research gaps.

Methodology. Systematic literature survey is the applied methodology. EBSCO and Taylor \& Francis database were used as a source for the sampling process.

Key findings. The analysis does acknowledge the need for more qualitative and rigorous research in this field and brings several recommendations for the future studies. The main conclusions also do urge for a more diversified research in terms of the sectors discussed, as the biggest gap identified is in the services sector. There is also a need for a more structured classification of the measures, depending on the real research focus: $\mathrm{CE}$ antecedents or outcomes.

Keywords: corporate entrepreneurship, intrapreneurship, corporate venturing.

JEL Codes: o31, o32.

\footnotetext{
* Faculty of Management and Economics, Gdańsk University of Technology. E-mail: mpop@pg.edu.pl.
} 


\section{INTRODUCTION}

Corporate entrepreneurship (CE) is a concept which has been present, under various names, in the literature for more than thirty years. During this period, in connection with the globalization process, with the evolution of the management science, it underwent a constant evolution of its definition and domain. The very term itself frequently overlaps with others such as intrapreneurship or corporate venturing. Scholars and researchers have not defined CE consistently (Entebang \& Harrison, 2012). Trying to understand and study $\mathrm{CE}$ in the national or international perspective is a complex issue for the researcher.

Surely, one cannot analyze CE in isolation from entrepreneurship theory, as it is just an extension of this concept to be used in describing entrepreneurial processes taking place within existing organizations. Schumpeter (1934), the father of innovation and entrepreneurship, argued that the entrepreneurial process involves new combinations, and in particular, doing new things or doing things in a new way. New combinations were enabling the entrepreneur to introduce new goods, new production methods, opening new markets, new supply sources, or to fund new organizations. Later, this innovation capacity has been assigned also to organizations and not only to individuals any more. Sharma and Chrisman (1999) treat entrepreneurship as an organizational creation, renewal or innovation that occurs within an existing organization or outside of it. The following discussion clarifies and establishes $\mathrm{CE}$ as an activity of the firm.

In Poland, CE is rather poorly researched and studied. Only few scholars decided to analyze and measure CE outcomes and antecedents. Therefore, the author decided to make a review of the theoretical or empirical literature using a research sample based on two the most important databases. The most important aim of this conceptual study is to identify the existing gaps and propose some recommendations for the future research. To achieve this goal, around 70 papers were selected (with the timeline 1996-2016) for further analysis, beginning with the effort of clarification of definitional issues, mentioned by numerous scholars. This allowed us to study the $\mathrm{CE}$ domain and to attempt the categorization of the selected articles, through a critical analysis of their outcomes. The conclusions 
indicate some directions for future research within a rather wide, going beyond one country, framework.

\section{THEORETICAL BACKGROUND}

Corporate entrepreneurship (CE) is a notion used to describe entrepreneurship occurring in a mid to large sized organizations (Morris et al., 2008). Entrepreneurship of organizations is a multi-component construct and plays a central role in the enhancement of organizational performance (Dyduch, 2008). According to Kuratko et al. (1990), the need to pursue CE has arisen from different problems faced by the enterprises in the turbulent environment: (1) required changes, innovations, and improvements in the marketplace to avoid stagnation and decline (Miller \& Friesen, 1982); (2) perceived weakness in the traditional methods of corporate management, and (3) the turnover of innovative-minded employees who are disenchanted with bureaucratic organizations. Schindehutte et al. (2000) generated a list of events stimulating or triggering an engagement to $\mathrm{CE}$, grouped later on into five categories (Kuratko et al., 2004):

1. Internal/external source,

2. Opportunity-driven/threat-driven,

3. Technology-pushed/market pull,

4. Top down/bottom up,

5. Systematic or deliberate search/chance or opportunism.

$\mathrm{CE}$ may be also viewed as consisting of two types of phenomena and processes surrounding them: (1) the birth of new businesses within existing organizations, whether through internal innovation or venturing and (2) the transformation of organizations through strategic renewal, i.e. the creation of new wealth through the combination of resources (Guth \& Ginsberg, 1990, p. 5; Dess et al., 1999, p. 85).

Narayanan et al. (2009) state that CE focuses on the various steps and processes associated with creating new businesses and integrating them into the firm's overall business portfolio. CE activities can be internally or externally oriented. The internal activities include product, process and administrative innovations at different levels of the organization (Zahra, 1991). Internal (or intra-corporate) entrepreneurship refers to all formalized entrepreneurial activities 


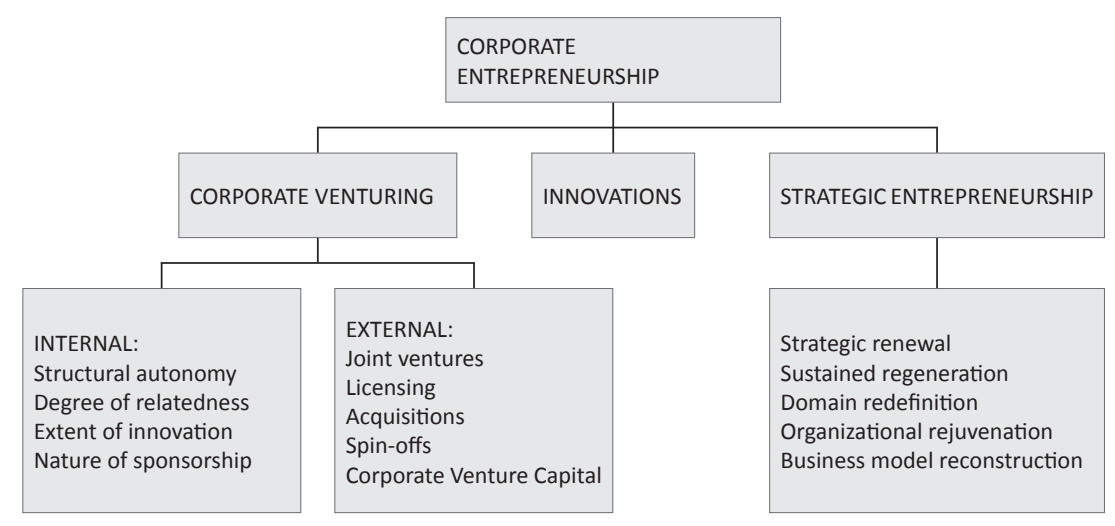

Figure 1. The corporate entrepreneurship concept content

Source: own understanding based on: Sharma \& Chrisman, 1999; Covin \& Miles, 1999; Kuratko \& Audretsch, 2009.

within existing business organizations. Formalized internal entrepreneurial activities are those which receive explicit organizational sanction and resource commitment for the purpose of innovative corporate endeavors - new product developments, product improvements, new methods or procedures (Schollhammer, 1982, p. 21).

External CE consists of combining resources dispersed in the environment by individual entrepreneurs with their unique resources to create a new resource combination independent of all others (Gautam \& Verma, 1997) and includes mergers, joint ventures, venture spin-offs and others.

The CE activities may be also of formal or informal character. Informal activities result from individual creativity or pursuit of self-interest, which after the formal recognition may become an integral part of the business. They aim at creating new business in established companies through product a process innovations and market developments (Zahra, 1991, p. 262). According to Michalski (2005), CE maybe differentiated into two dimensions: the degree of organizational separation from the core business of $\mathrm{CE}$ function and the degree of institutionalization of the $\mathrm{CE}$ function. The $\mathrm{CE}$ function is categorized into four main governance types: development of new products and services, corporate development, single corporate ventures, corporate venture portfolios. The two first are serving for resource exploitation and the two last to resource exploration. 
The corporate venturing (CV) is a means of planning organizational ambiguity in entrepreneurial action by separating one group of intrapreneurs from the organizational structure. It may be divided into internal and external CV (Sharma \& Chrisman, 1999). The internal CV results in creation of a new business within the existing corporate structure, while the external CV refers to activities that result in the creation of semi-autonomous or autonomous organizational entities that reside outside the existing organizational domain. New businesses created through CV may be heterogeneous in terms of their markets, products and innovativeness, as well as in terms of their parent incubator organizations (Phan et al., 2009).

Wolcott and Lippitz (2007) conceptualize four models of CE, including: the opportunist, the enabler, the advocate and the producer model. The framework is composed of two dimensions: organizational ownership (who within the organization has primary ownership for the creation of new business?) and resource authority (is there a dedicated "pot of money" allocated to CE?). In the opportunist model, CE is based on the efforts in spite of the corporation of project champions, while in the three following models, $\mathrm{CE}$ is actively managed.

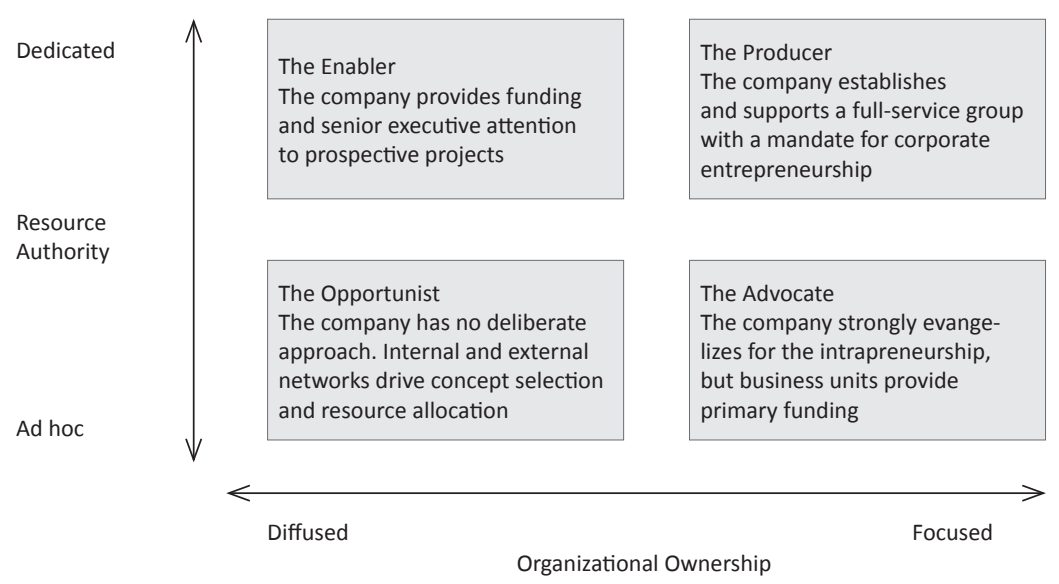

Figure 2. The four models of corporate entrepreneurship Source: Wolcott \& Lippitz, 2007, p. 77.

These two dimensions, under the direct control of management, differentiate how companies approach CE. In order to select the right model, companies need to articulate a strategic vision of 
growth, define and communicate company's objectives for $\mathrm{CE}$, and build corporate and divisional leadership. Authors recommend also to start with quick wins which will build credibility and then constantly adapt to the changing situation. The scope of $\mathrm{CE}$ becomes wider as organizations not previously recognized as entrepreneurial, need to change their approach in order to survive and succeed in the increasingly competitive environments (Phan et al., 2009). Obviously, the $\mathrm{CE}$ activities within corporations are heterogeneous and there is a need to know more about this variety (Narayanan et al., 2009). Authors claim that, depending on the ambitions and organizational capacity, the company has to select one of the proposed models. Each of them requires different forms of leadership, processes and skill sets. Recently, the most popular perspectives searching for better understanding of the CE phenomenon are: competence, knowledge, learning, cognition (Adenfelt \& Lagerström, 2006, Hayton \& Kelley, 2006; West, 2007) and network (Yiu \& Lau, 2008).

Referring to the scope of $\mathrm{CE}$, numerous scholars stress the role of management at different levels of the organization, especially of the top management leadership, shaping the internal organization of CE (Zahra et al., 2000; Dess et al., 2003). According to Ferreira (2005), CE depends on the employees at operational level and their capacity to exploit entrepreneurial opportunities. Effective leaders can provide the organization with better information about entrepreneurial opportunities and enable the entrepreneurial mindset among the staff members by the regular compensation of their skills and tacit knowledge and the tolerance for failure of ambitious, creative projects.

\section{RESEARCH METHOD}

Since this paper is a review of the theoretical or empirical literature dedicated to corporate entrepreneurship, the research sample was based on two main databases: EBSCO and Taylor \& Francis as the most representative for the management research. This research was performed in February 2017. The timespan for the research was 1996 to 2016. However, as the notion of CE has been continuously discussed for over three decades (sometimes under different names), 
some important sources preceding the initial time frame had to be analyzed. Several search paths with the following key words were used for the selection: corporate entrepreneurship, intrapreneurship, corporate venturing.

At first, 67 papers were selected based on abstracts and in the second phase, after a deeper review of the abstracts, only 58 papers were chosen for the further analysis. Then, it turned out that several papers contained other possibly related references, therefore, 19 additional articles were deemed as relevant for further review. During the snowballing review, 11 articles were chosen as relevant for further analysis. At this stage the sample of 69 papers was established for the main study, beginning with an effort of clarification of definitional issues, mentioned by numerous scholars.

In parallel, the analysis of the main components of the proposed $\mathrm{CE}$ constructs has been performed. The following were selected as coding criteria: publication year, type of article, definition of $\mathrm{CE}$, intrapreneurship or corporate venturing, type of studied organization/firm, measures or attributes of CE, research methods and country, the study was performed for. This approach enabled the author to propose an attempt to conceptualize CE and identify the existing research gaps, and, consequently, to make recommendations regarding the next research directions.

\section{RESULTS AND DISCUSSION}

The concept of corporate entrepreneurship was introduced few decades ago and evolved from the notion of "intrapreneurship", which was popularized by Macrae (1976, 1982) and Pinchot (1985), who identified intrapreneurs as "in house entrepreneurs, those dreamers who can increase the speed and cost-effectiveness of technology transfer from R\&D to the marketplace" (1985, p. 14). Hostager et al. (1998) understand them as individuals and groups working within corporation to (1) identify ideas for new products or services (2) turn these ideas into profitable products or services (1998, p. 11-12). Thornberry (2003, p. 331) describes intrapreneurs as those who bring to bear the mindset and behaviors characteristics of external entrepreneurs and transpose them to an existing and usually large corporate setting. In parallel, there is a strong stream of research 
oriented toward another, very important aspect of entrepreneurial behavior of the organizations - a corporate venturing (CV). Von Hippel (1977) defined it as an activity that seeks to generate new businesses for the corporation in which it resides through the establishment of external or internal corporate ventures (p. 163). This activity, involving the creation of the new venture within an existing business unit (Zajac et al., 1991; Hornsby et al., 1993). Both concepts do not exclude one another, they are rather complementary, and in my opinion, provide a more holistic construct of a corporate entrepreneurship. At the same time, in the literature both of them evolve independently and in parallel with other terms describing entrepreneurial orientation of an organization. Additionally, there is an important number of definitions of the main overlapping notions of intrapreneurship, entrepreneurial orientation, corporate venturing, strategic entrepreneurship and corporate entrepreneurship. It clearly reveals that the corporate entrepreneurship construct is still evolving, not only through contributions of scholars, but also within the work of business practitioners. In their conceptualizations through the last three decades they were proposing numerous definitions of $\mathrm{CE}$, sometimes hidden under the other, aforementioned terms.

Therefore, the author decided to select some of the definitions to better understand and investigate the evolution of this concept. For the sake of further research and conceptualization effort, the journey through constructs and their definitions was accompanied with an attempt at looking for measures/attributes, research method used and the country of research (see: Table 1). As it may be easily noticed, the same term is sometimes used differently by various authors, and some of them use diverse terms to describe the same phenomenon (Sharma \& Chrisman, 1999). Some constructs, in particular corporate venturing, are also studied as an element or one of the three possible components of the CE meta-construct (see: Figure 1). All this confirms that significant complexity and conceptual confusion remains in the field and the need for clarification is still important. 


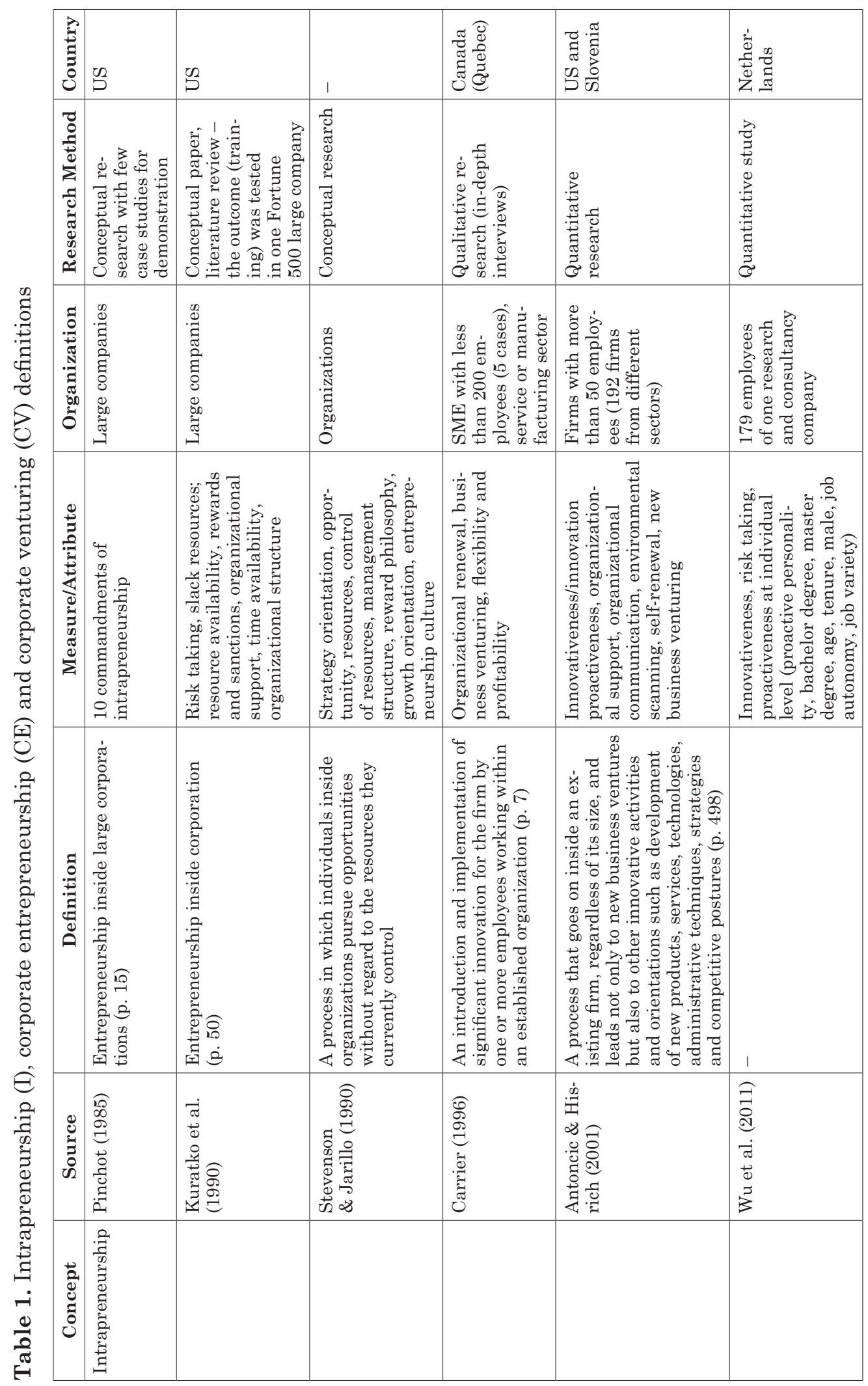




\begin{tabular}{|c|c|c|c|c|c|c|c|c|}
\hline 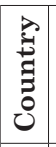 & & 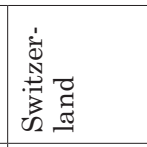 & $\stackrel{n}{2}$ & 1 & $\frac{1}{5}$ & 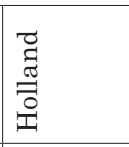 & 1 & $y^{2}$ \\
\hline & 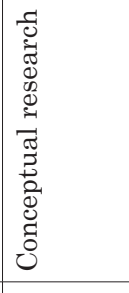 & 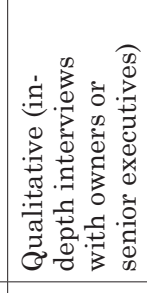 & 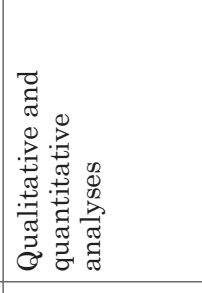 & 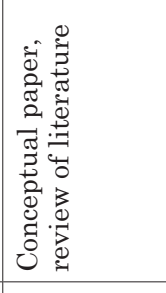 & 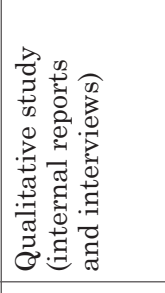 & 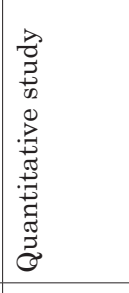 & 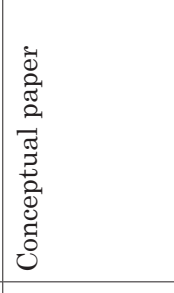 & 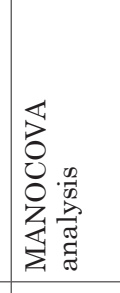 \\
\hline 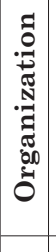 & 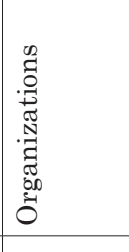 & 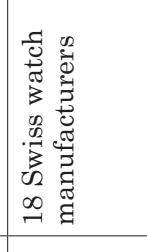 & 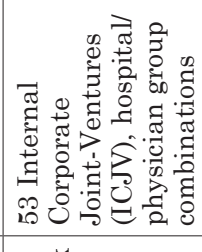 & 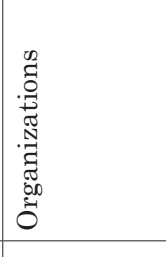 & 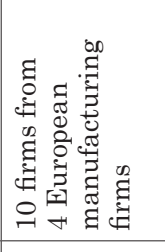 & 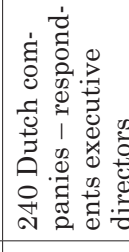 & 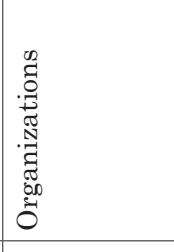 & \\
\hline & 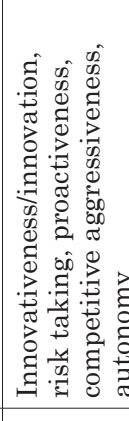 & 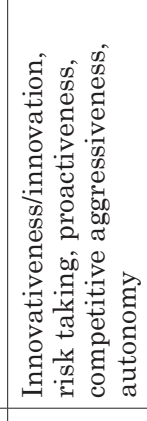 & 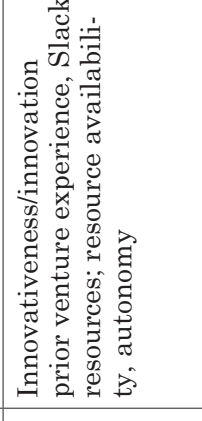 & 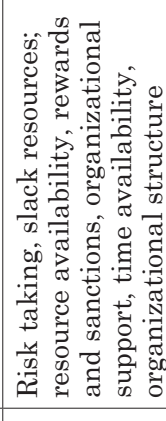 & 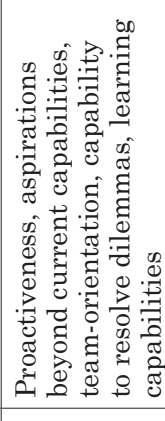 & 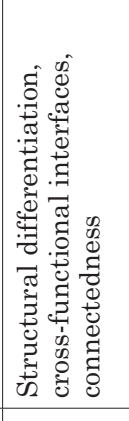 & 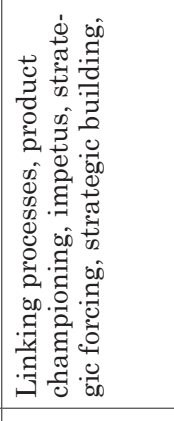 & 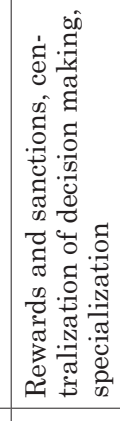 \\
\hline & 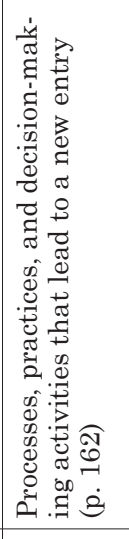 & 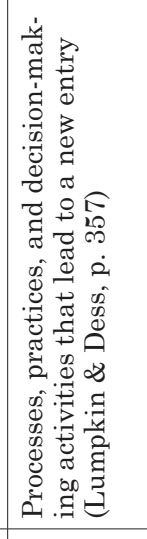 & 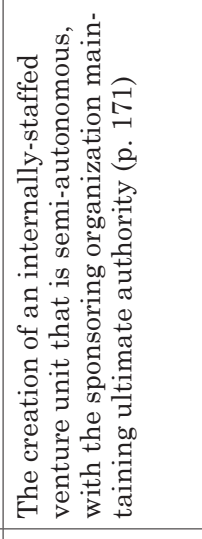 & 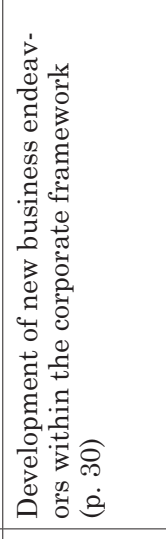 & 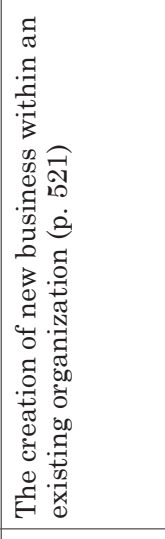 & 1 & 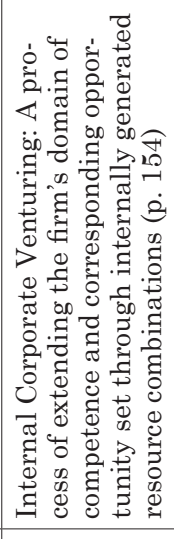 & 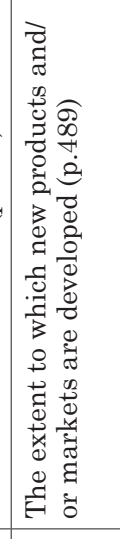 \\
\hline & 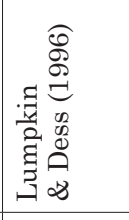 & 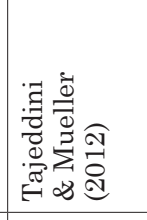 & 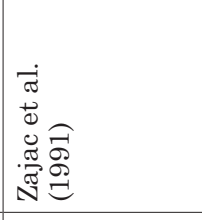 & 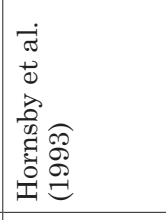 & 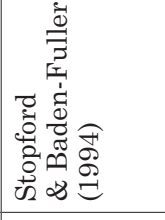 & 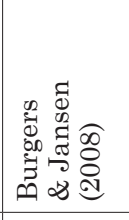 & 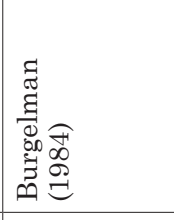 & 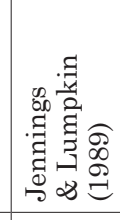 \\
\hline & 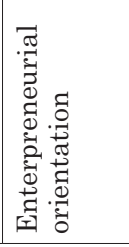 & & 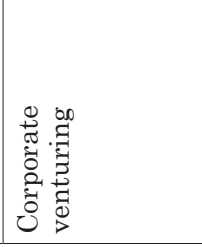 & & & & 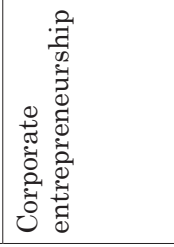 & \\
\hline
\end{tabular}




\begin{tabular}{|c|c|c|c|c|c|c|c|}
\hline & 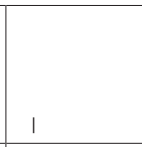 & 1 & 1 & $\stackrel{n}{5}$ & 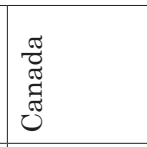 & 1 & 1 \\
\hline & 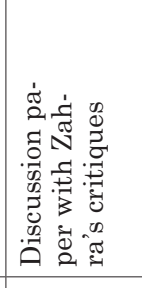 & 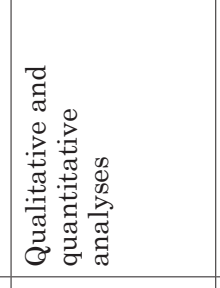 & 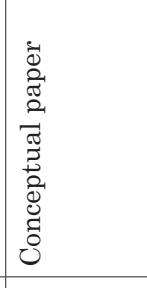 & 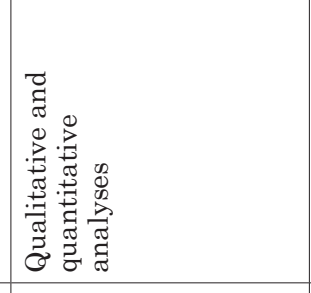 & 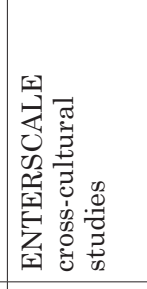 & 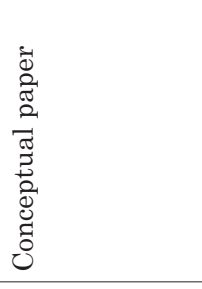 & 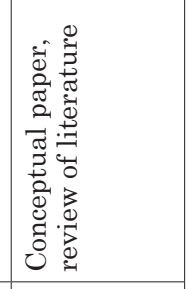 \\
\hline & ". & 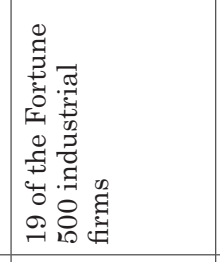 & 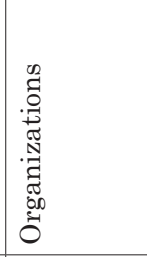 & 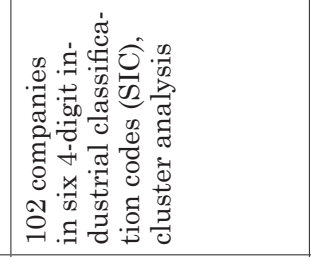 & 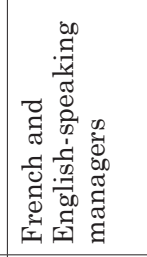 & 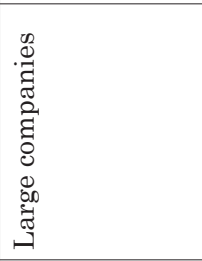 & 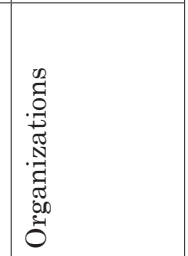 \\
\hline 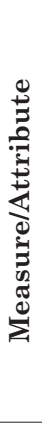 & 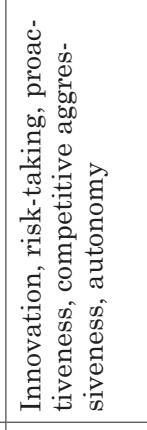 & 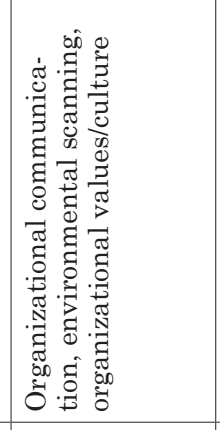 & 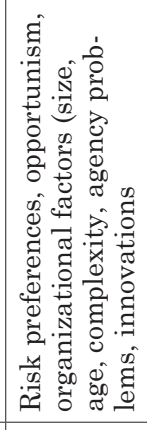 & 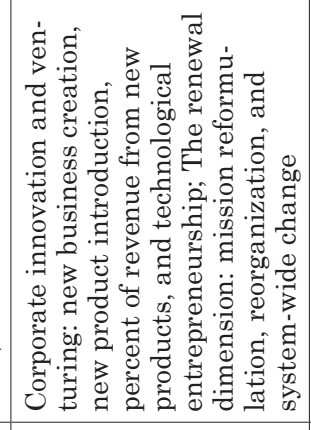 & 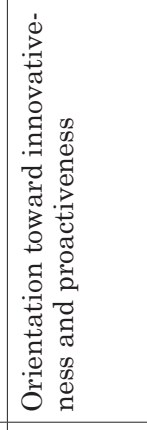 & 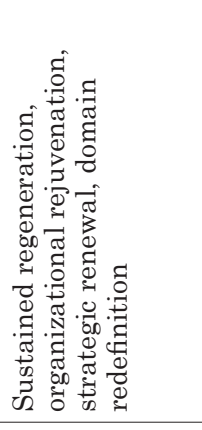 & 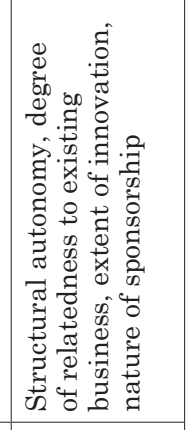 \\
\hline 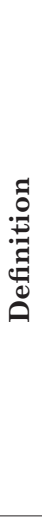 & 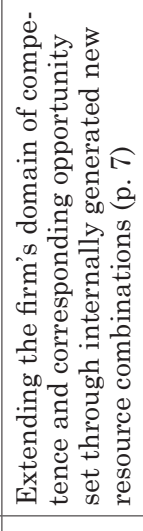 & 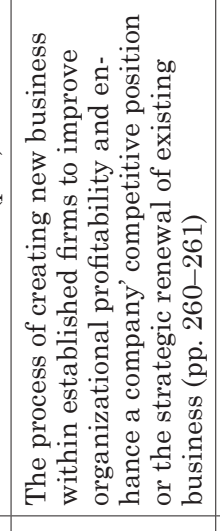 & 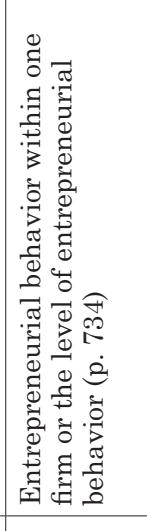 & 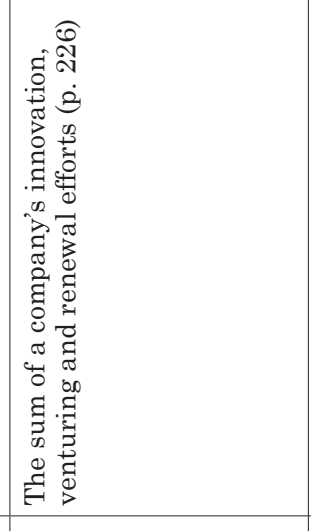 & 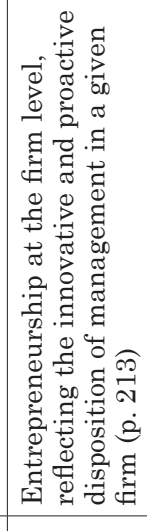 & 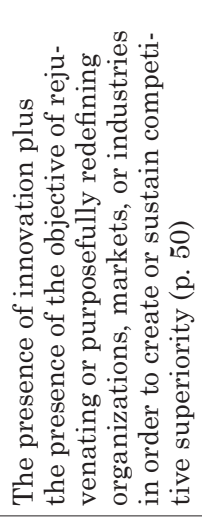 & 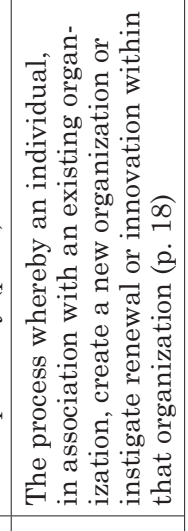 \\
\hline 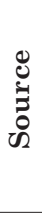 & 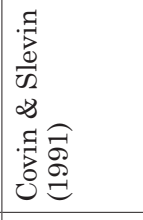 & 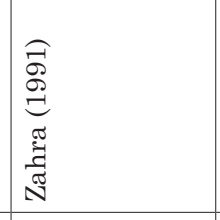 & 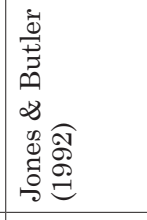 & 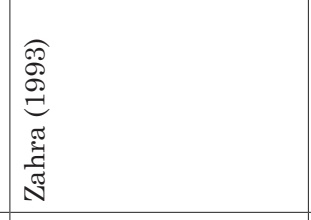 & 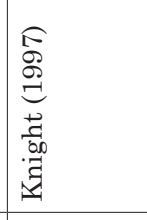 & 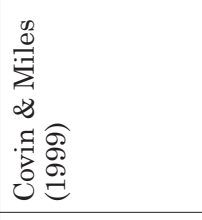 & 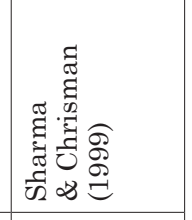 \\
\hline & & & & & & & \\
\hline
\end{tabular}




\begin{tabular}{|c|c|c|c|c|c|c|}
\hline & 2 & $\stackrel{20}{5}$ & 1 & $\stackrel{20}{5}$ & 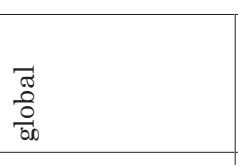 & 1 \\
\hline 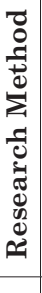 & 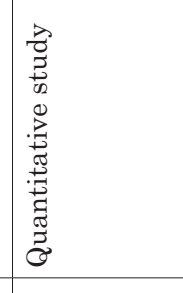 & 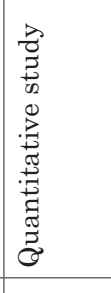 & 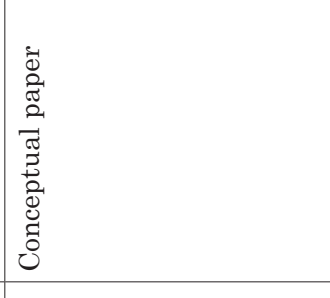 & 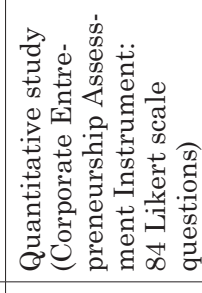 & 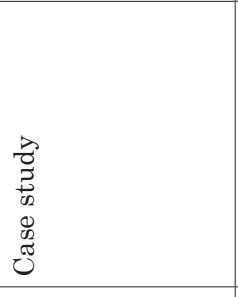 & 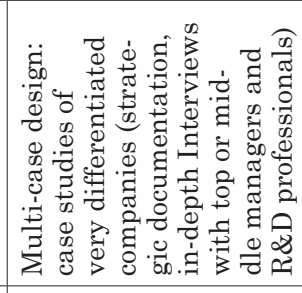 \\
\hline & 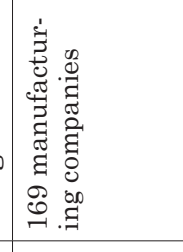 & 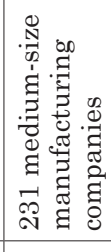 & 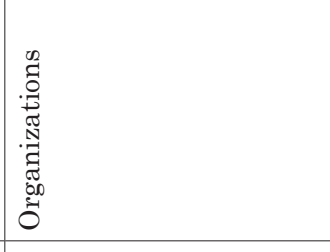 & 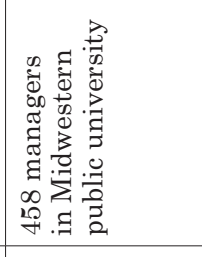 & 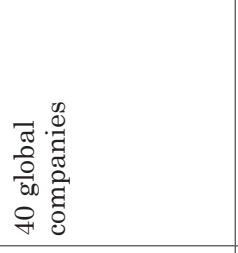 & 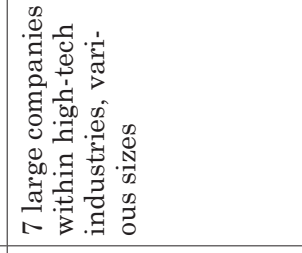 \\
\hline 苋 & 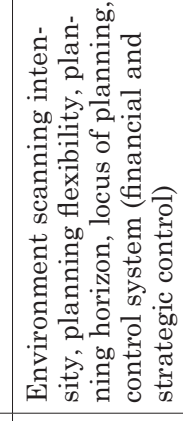 & 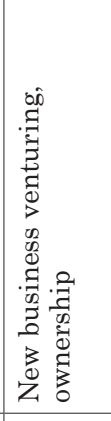 & 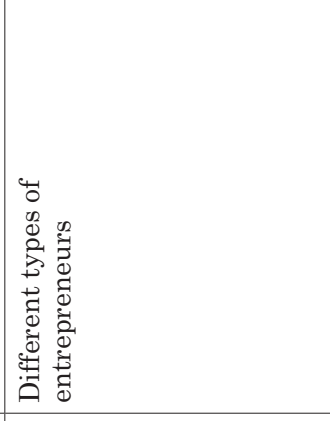 & 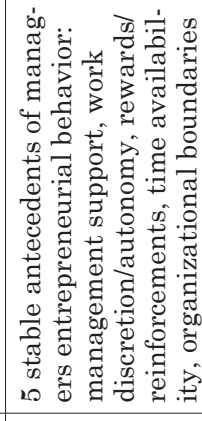 & 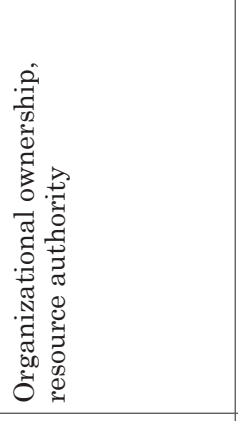 & 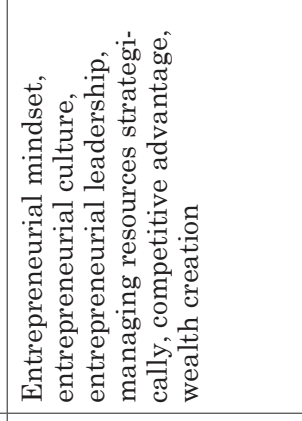 \\
\hline : & & 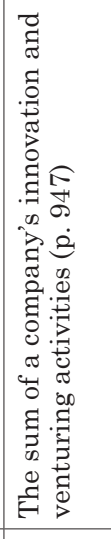 & 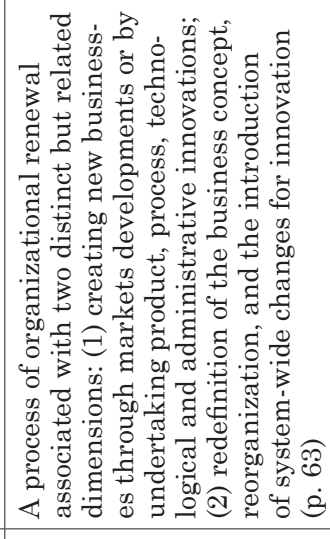 & 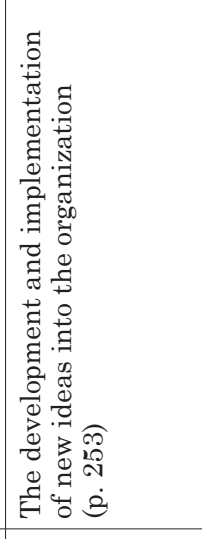 & 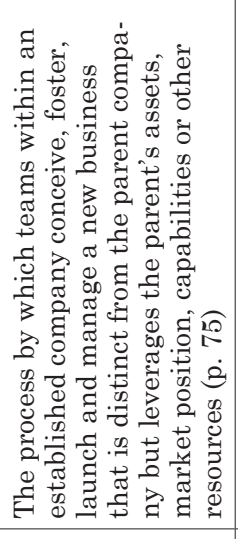 & 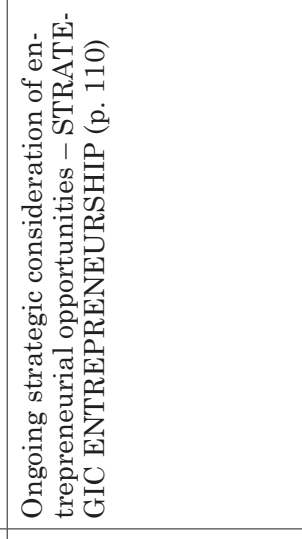 \\
\hline 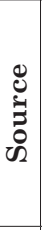 & 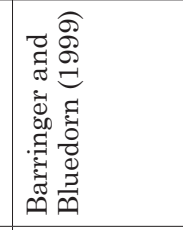 & 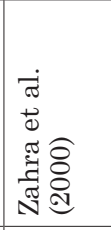 & 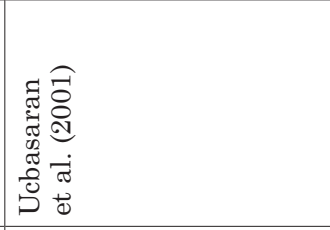 & 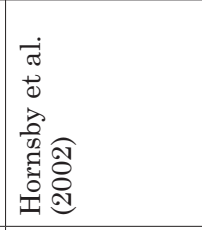 & 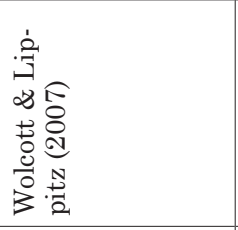 & 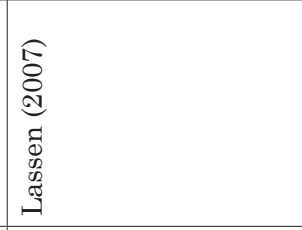 \\
\hline 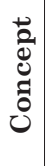 & & & & & & \\
\hline
\end{tabular}




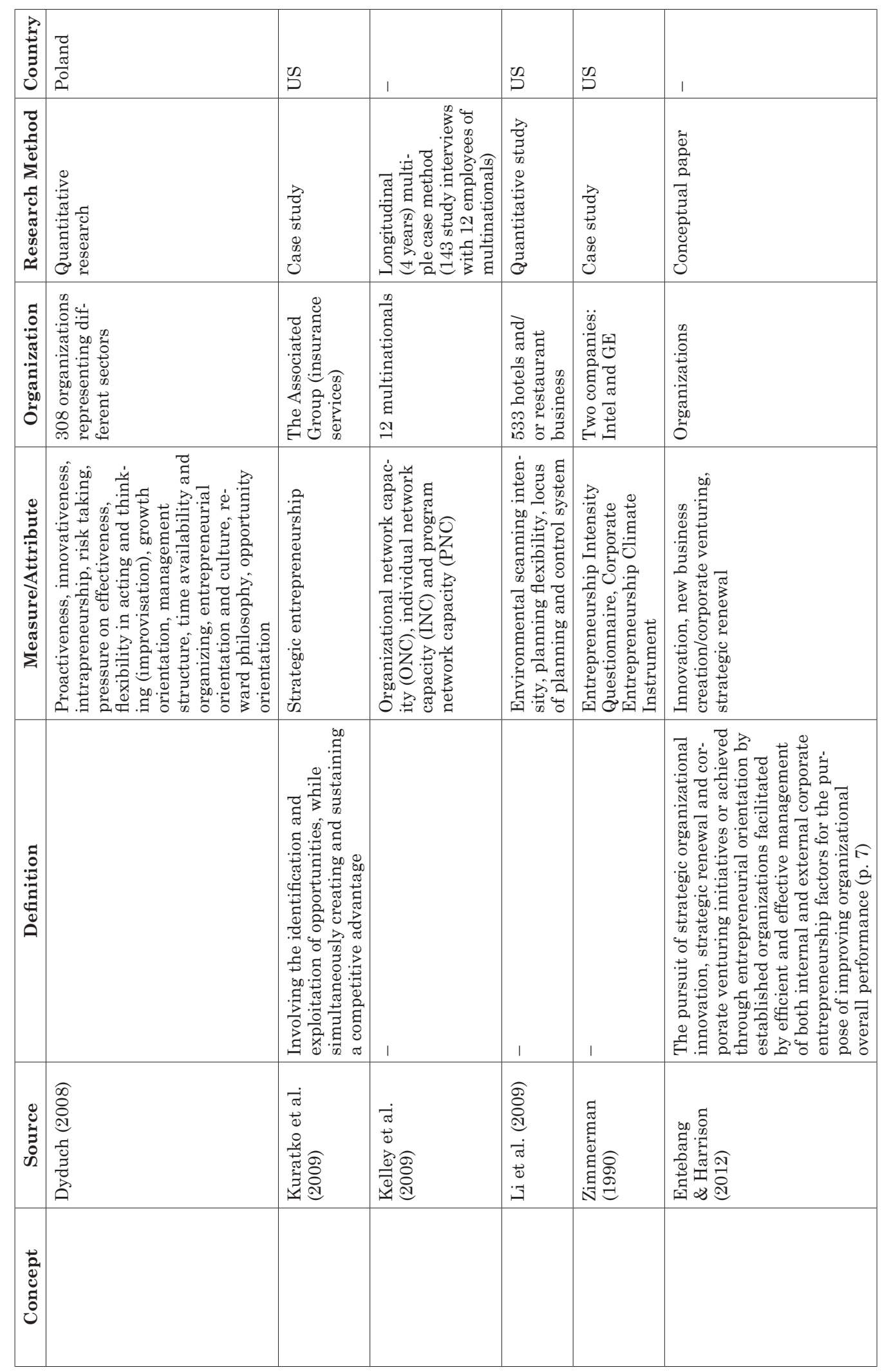




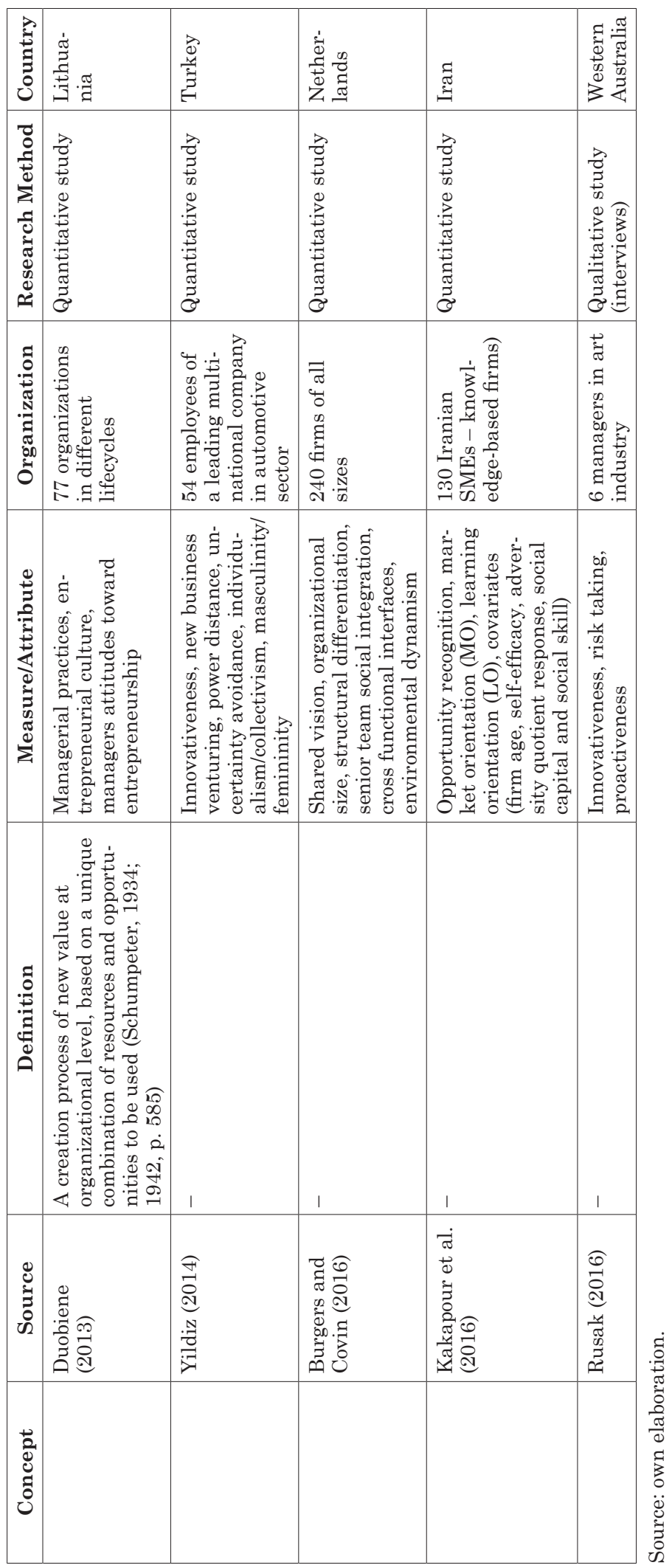


As presented above (see: Table 1), most of the scholars have tried to identify the factors that encourage the $\mathrm{CE}$ within an existing organization for more than three decades. Some authors did not propose their own definition of $\mathrm{CE}$, simply applying already existing in the literature delimitation, notwithstanding, these studies offered an interesting input in terms of the other analyzed elements (measures, organization size, research methods, or country). The outcome of this comparison clearly shows the confusion in the use of the presented constructs and deployment of very similar measures for describing all of them. Some of the presented studies allowed their authors for proposing a relatively sophisticated tools for measuring the corporate entrepreneurship activity, mostly based on questionnaires addressed to the organization's managers (see: Table 2). This, according to the author, partially explains the recurring use of methods by different generations of scholars. These tools have been validated in widely diversified organizations both in terms of sector and size.

Table 2. Main tools for measuring CE

\begin{tabular}{|l|l|l|}
\hline \multicolumn{1}{|c|}{ Tool } & \multicolumn{1}{|c|}{ Topic } & \multicolumn{1}{c|}{ Elements of the tool } \\
\hline $\begin{array}{l}\text { ENTRESCALE (Miller } \\
\text { \& Friesen, 1992; Covin } \\
\text { \& Slevin, 1999; Knight, } \\
\text { 1997) }\end{array}$ & $\begin{array}{l}\text { Assessment of } \\
\text { the firm's entrepreneurship }\end{array}$ & $\begin{array}{l}\text { Environmental hostility, } \\
\text { organization structure, stra- } \\
\text { tegic posture and financial } \\
\text { performance }\end{array}$ \\
\hline $\begin{array}{l}\text { Innovation-venturing-renew- } \\
\text { al scale (Zahra, 1996) }\end{array}$ & $\begin{array}{l}\text { Entrepreneurial activities of } \\
\text { the organization }\end{array}$ & $\begin{array}{l}\text { Innovation, venturing } \\
\text { renewal }\end{array}$ \\
\hline $\begin{array}{l}\text { Entrepreneurial Orientation } \\
\text { Scale (Lumpkin \& Dess, } \\
\text { 1996) }\end{array}$ & $\begin{array}{l}\text { The ways in which enter- } \\
\text { prise combine and transform } \\
\text { tangible resources }\end{array}$ & $\begin{array}{l}\text { Innovativeness/innovation, } \\
\text { risk taking, proactiveness, } \\
\text { competitive aggressiveness, } \\
\text { autonomy }\end{array}$ \\
\hline $\begin{array}{l}\text { Entrepreneurial Perfor- } \\
\text { mance Index (EPI) (Morris } \\
\text { \& Sexton, 1996) }\end{array}$ & $\begin{array}{l}\text { Level of intensity of entre- } \\
\text { preneurship in organizations }\end{array}$ & $\begin{array}{l}\text { Innovativeness, risk taking, } \\
\text { proactiveness }\end{array}$ \\
\hline $\begin{array}{l}\text { Corporate Entrepreneurship } \\
\text { Assessment Instrument } \\
\text { (CEAI) (Hornsby et al., 1993; } \\
\text { Morris \& Kuratko, 2002) }\end{array}$ & $\begin{array}{l}\text { Organizational factors } \\
\text { to help managers (leaders) } \\
\text { focus their effort to encour- } \\
\text { age CE }\end{array}$ & $\begin{array}{l}\text { Management support, work } \\
\text { discretion, rewards and rein- } \\
\text { forcement, time availability, } \\
\text { organizational boundaries }\end{array}$ \\
\hline $\begin{array}{l}\text { Entrepreneurial Manage- } \\
\text { ment (Stevenson \& Jarillo, } \\
\text { 1990; Brown et al., 2001) }\end{array}$ & $\begin{array}{l}\text { Entrepreneurship as } \\
\text { the question of strategic } \\
\text { management }\end{array}$ & $\begin{array}{l}\text { Strategy orientation, oppor- } \\
\text { tunity, resources, control } \\
\text { of resources, management } \\
\text { structure, reward philos- } \\
\text { ophy, growth orientation, } \\
\text { entrepreneurship culture }\end{array}$ \\
\hline
\end{tabular}

Source: own description based on Scheepers et al., 2007; Sakhdari, 2016. 
The existence of these tools certainly enhances the quantitative approach to study $\mathrm{CE}$ as the majority of them, besides scientific aims, have direct practical applications. The CEAI, for instance, measures entrepreneurial behaviors at the individual level - this analysis may help the organization to identify the factors which may help her to reach higher and higher levels of entrepreneurial attitude. Unfortunately, as with most scales developed in North America, some of the tools still lack strong evidence of cross-cultural validity, reliability and freedom from cultural bias. Indeed, some but still not numerus attempts have been made to thoroughly assess the scales' psychometric properties in cross-cultural studies. This effort needs to be continued (Sakhdari, 2016).

Although empirical evidence related to $\mathrm{CE}$ has been around for more than three decades, it remains fairly ambiguous. Hence, different scholars have used diversified perspectives to approach $\mathrm{CE}$ and all categorization effort seems very challenging.

Entrepreneurship researchers appear to perceive CE as an entrepreneurial activity, a process, a strategy and a behavior executed by a group of employees in existing organizations for the purpose of creating structural growth and improving competitive position through innovation, strategic renewal, and corporate venturing activities (Entebang \& Harrison, 2012), or analyzed at different levels, such as organizational, venture or individual levels (Belousova et al., 2010). At the organizational level, numerous papers talk about the model of corporate entrepreneurial strategy (CES) (Ireland et al., 2009; Kreiser et al., 2011; Kearney \& Meynhardt, 2016), entrepreneurial orientation (EO) (Covin et al., 2005; Lumpkin \& Dess, 1996), entrepreneurial management (Brown et al., 2001; Stevenson \& Jarillo, 1990), firm behavior (Antoncic \& Hisrich, 2003), the organizational learning perspective (Dess et al., 2003; Sambrook \& Robert, 2005); wealth creation (Antoncic \& Hisrich, 2004); a competency-based perspective (Hayton \& Kelley, 2006); the human resource approach (Maes et al., 2005). At the venture or project level, researchers investigate the evolution of the idea into a final product (Burgelman, 1984; Vesper, 1984). At individual level, scholars focus on entrepreneurial individuals within organization (Pinchot, 1985; Jones \& Butler, 1992).

The other classification may be organized according to t; he purpose of analysis of $\mathrm{CE}$ phenomenon. In this approach, one can distinguish studies aiming at conceptualizing this phenomenon and 
making a review of the existing research evidence, a large group of articles analyzing the antecedents of $\mathrm{CE}$ and a few less numerous articles on its outcomes. The selected papers, grouped in the thematic associations mentioned above may be divided into some conceptual subgroups (see: Table 3). Regarding the antecedents, three main concepts emerge from the analysis: environment, firm and management team, somehow dissociated from the enterprise itself. Regarding the effects of $\mathrm{CE}$, the main research focus is on the growth, competitiveness, performance and innovation. Although, such a categorization may be questionable as it is difficult to separate performance from competitiveness, innovation and growth, it constitutes the first attempt of the author, based on the performed analysis.

Table 3. Looking for the CE research categorization

\begin{tabular}{|c|c|c|}
\hline $\begin{array}{c}\text { Conceptualization and } \\
\text { reviews }\end{array}$ & Antecedents of CE & CE outcomes \\
\hline $\begin{array}{l}\text { domain of CE (Zahra, 1996, } \\
\text { Sharma \& Chrisman, 1999; } \\
\text { Covin \& Miles, 1999; Kurat- } \\
\text { ko \& Audretsch, 2009) }\end{array}$ & $\begin{array}{l}\text { environment (Simsek } \\
\text { \& Heavey (2011) Romero- } \\
\text { Martínez et al., 2010; Zahra, } \\
\text { 1991, 1993) }\end{array}$ & $\begin{array}{l}\text { growth (Antoncic \& Hisrich, } \\
2001 \text { ) }\end{array}$ \\
\hline $\begin{array}{l}\text { implementation of CE (Wol- } \\
\text { cott \& Lippitz, 2007) }\end{array}$ & $\begin{array}{l}\text { firm (Behrens \& Patzelt, } \\
\text { 2016; Kellermanns \& Edd- } \\
\text { leston, 2006; Nason et al., } \\
\text { 2015; Simsek et al., 2009; } \\
\text { Yiu \& Lau, 2008) }\end{array}$ & $\begin{array}{l}\text { competitiveness (Hitt et al., } \\
\text { 2001; Paunović, 2012) }\end{array}$ \\
\hline $\begin{array}{l}\text { literature review (Phan } \\
\text { et al., 2009; Höglund, 2009; } \\
\text { Corbett et al., 2013; Sakh- } \\
\text { dari, 2016) }\end{array}$ & $\begin{array}{l}\text { management team (Zahra } \\
\text { et al., 2000; Dess et al., } \\
\text { 2003; Hayton \& Kelley, } \\
\text { 2006; Heavey \& Simsek, } \\
\text { 2013; Ling et al., 2008; Naldi } \\
\text { et al., 2015; Behrens \& Pat- } \\
\text { zelt, 2016) }\end{array}$ & $\begin{array}{l}\text { performance (Zahra, } \\
\text { 1996; Rauch et al., 2009; } \\
\text { Dyduch, 2008; Obłój et al., } \\
\text { 2010; Engelen et al., 2012; } \\
\text { Bratnicka \& Bratnicki, 2013, } \\
\text { Żur, 2013) }\end{array}$ \\
\hline $\begin{array}{l}\text { measures (Maes, 2003; } \\
\text { Wójcik-Karpacz, 2016) }\end{array}$ & & $\begin{array}{l}\text { innovation (Barringer } \\
\& \text { Bluedorn, 1999; } \\
\text { McFadzean et al., 2005; } \\
\text { Heidemann Lassen, Gertsen } \\
\text { \& Riis, 2006) }\end{array}$ \\
\hline $\begin{array}{l}\text { entrepreneurial manage- } \\
\text { ment (Stevenson \& Jarillo, } \\
\text { 1990) }\end{array}$ & & \\
\hline
\end{tabular}

Source: own elaboration.

This approach to the structuring the most important literature enabled us to arrive at some conclusions and, at the same time, recommendations for the further research. 


\section{CONCLUSIONS}

Researchers in corporate entrepreneurship use very wide scope of perspectives for its analysis. The most important seems the corporate venturing $(\mathrm{CV})$ perspective, conceived on the one hand, as an integral part of $\mathrm{CE}$, and on the other hand, as a much broader construct of CE understood as internal corporate venturing (Burgelman, 1984). The greatest number of conceptual studies has been conducted in the Anglo-Saxon countries, which has changed only in the recent years due to Chinese and West-European researchers. Consequently, the main theoretical and empirical evidence come from rather similar economic environment and business culture. The evidence from young market economies is missing.

Table 4. Summary of the main conclusions et recommendations

\begin{tabular}{|l|l|l|}
\hline \multicolumn{1}{|c|}{ Research outcome } & \multicolumn{1}{|c|}{ Criticalities } & \multicolumn{1}{c|}{ Recommendation } \\
\hline $\begin{array}{l}\text { The empirical evidence dominat- } \\
\text { ed by US, few Chinese and Euro- } \\
\text { pean papers (mainly from WE) }\end{array}$ & $\begin{array}{l}\text { Business culture } \\
\text { Economic context }\end{array}$ & $\begin{array}{l}\text { Need for more country } \\
\text { specific studies }\end{array}$ \\
\hline $\begin{array}{l}\text { Predominance of quantitative } \\
\text { methods }\end{array}$ & $\begin{array}{l}\text { Sample } \\
\text { Quality of respondents }\end{array}$ & $\begin{array}{l}\text { Need for more qualitative } \\
\text { approach }\end{array}$ \\
\hline $\begin{array}{l}\text { Predominance of evidence from } \\
\text { manufacturing sector }\end{array}$ & $\begin{array}{l}\text { Innovation pressure } \\
\text { R\&D }\end{array}$ & $\begin{array}{l}\text { Need for sector specific } \\
\text { evidence }\end{array}$ \\
\hline $\begin{array}{l}\text { Little relevance with the size of } \\
\text { firms }\end{array}$ & $\begin{array}{l}\text { Generalization of } \\
\text { outcomes }\end{array}$ & $\begin{array}{l}\text { Need for size specific } \\
\text { evidence }\end{array}$ \\
\hline Multiplicity of measures of CE & Irrelevance of application & $\begin{array}{l}\text { Need for classification of } \\
\text { measuring tools }\end{array}$ \\
\hline
\end{tabular}

Source: own elaboration.

The predominance of quantitative research methods is visible. Qualitative research methods, e.g., case studies, are much less popular, especially in the first twenty years of CE research. Therefore, there is a need for more qualitative or mixed research methods.

Additionally, the manufacturing sector has been predominating. Considering that this sector is the most exposed to the pressure of innovation, quite commonly equipped with B\&R units, so also the most committed to the implementation of new organizational approaches aiming at enhancing the innovativeness, there is certainly a necessity of more diversified sectoral research. The service sector, the most developing among others worldwide in recent years has not yet received special attention from researchers. 
Another conclusion is related to the size of the organizations that have been studied. Contrary to the expectations of the author, the research was carried out not exclusively in corporations, constituting the background for evolution of the discussed construct, but also in small or micro enterprises. To allow any comparisons, there is a need for more organized size specific evidence.

There is also a multitude of tools for measuring CE, on the one hand applied to estimation of the antecedents and on the other of the CE outcomes. Researchers use the meta-analysis tools, organized around innovation-venturing-renewal. Therefore, the need for a more structured classification of CE measures and of their application, is evident.

Despite suggesting several recommendations, this paper comes with certain limitations. It provides quite a general overview of the corporate entrepreneurship phenomenon. No discussion regarding more specific aspects or nationally explored characteristics of $\mathrm{CE}$ has started yet. Thus, this article is only a starting point for more advanced research in an international or national perspective.

\section{REFERENCES}

Adenfelt, M. \& Lagerström, K. (2006). Organizational rejuvenation for knowledge exploitation: Exploring corporate entrepreneurship in an MNE. Journal of International Entrepreneurship, 4(2), 83-98.

Antoncic, B. \& Hisrich, R.D. (2001). Intrapreneurship: Construct refinement and cross-cultural validation. Journal of Business Venturing, 16(5), 495-527.

Antoncic, B. \& Hisrich, R.D. (2003). Privatization, corporate entrepreneurship and performance: Testing a normative model. Journal of Developmental Entrepreneurship, 8, 197-218.

Antoncic, B. \& Hisrich, R.D. (2004). Corporate entrepreneurship contingencies and organizational wealth creation. Journal of Management Development, 23(6), 518-550.

Baden-Fuller, C. (1995). New directions for effective strategy research. British Journal of Management, 6, 3-16.

Barrett, H. \& Weinstein, A. (1998). The effect of market orientation and organizational flexibility on corporate entrepreneurship. Entrepreneurship Theory and Practice, 23(1), 57-70.

Barringer, B.R. \& Bluedorn, A.C. (1999). The relationship between corporate entrepreneurship and strategic management. Strategic Management Journal, 20, 421-444. 
Behrens, J. \& Patzelt, H. (2016). Corporate entrepreneurship managers' project terminations: Integrating portfolio-level, individual-level, and firm-level effects. Entrepreneurship Theory and Practice, 40(4), 815-842.

Belousova, O., Gailly, B. \& Basso, O. (2010). A conceptual model of corporate entrepreneurial behavior, CRECIS, Working paper 06, Bruxelles: Louvain School of Management.

Bratnicka, K. \& Bratnicki, M. (2013). Linking two dimensions of organizational creativity to firm performance: The mediating role of corporate entrepreneurship and the moderating role of the environment. Advances in Business-Related Scientific Research Journal (ABSRJ), 4(2), 153-163.

Bratnicka, K. \& Kulikowska-Pawlak, K. (2016). Towards an Integrated Framework for Corporate Entrepreneurship. Problemy Zarzadzania, vol. 14, $3(62)$, t. 2, 24-35.

Brown, T.E., Davidsson, P. \& Wiklund, J. (2001). An operationalization of Stevenson's conceptualization of entrepreneurship as opportunity-based firm behavior. Strategic Management Journal, 22, 953-968.

Burgelman, R.A. (1984). Designs for corporate entrepreneurship. California Management Review, 26, 154-166.

Burgers, J.H. \& Covin, J.G. (2016). The contingent effects of differentiation and integration on corporate entrepreneurship. Strategic Management Journal, 37, 521-540.

Burgers, H. \& Jansen, J. (2008). Organizational ambidexterity and corporate entrepreneurship: The differential effects on venturing, innovation and renewal processes. In: A. Zacharakis (ed.). Frontiers of Entrepreneurship Research 2008: Proceedings of the $28^{\text {th }}$ Annual Entrepreneurship Research Conference (pp. 1-15). North Carolina, Chapel Hill: Babson College.

Carrier, C. (1996). Intrapreneurship in small business: An exploratory study. Entrepreneurship Theory and Practice, 21(1), 5-20.

Corbett, A., Covin, J.G., O'Connor, G.C. \& Tucci, C.L. (2013). Corporate entrepreneurship: State-of-the-art research and a future research agenda. The Journal of Product Innovation Management, 30(5), 812-820.

Covin, J.G. \& Miles, M.P. (1999). Corporate entrepreneurship and the pursuit of competitive advantage. Entrepreneurship Theory and Practice, 23(3), 47-63.

Covin, J.G. \& Slevin, D.P. (1991). A conceptual model of entrepreneurship as firm behavior. Entrepreneurship Theory and Practice, 16(1), 7-26.

Covin, J.G., Green, K.M. \& Slevin, D.P. (2005). Strategic process effects on the entrepreneurial orientation-sales growth rate relationship. Academy of Management Annual Meeting Proceedings, 1, K1-K6. 
Dess, G.G., Ireland, R.D., Zahra, S.A., Floyd, S.W., Janney, J.J. \& Lane, P.J. (2003). Emerging issues in corporate entrepreneurship. Journal of Management, 29(3), 351-378.

Dess, G.G., Lumpkin, G.T. \& McGee, J.E. (1999). Linking corporate entrepreneurship to strategy, structure, and process: suggested research directions. Entrepreneurship Theory and Practice, 23(3), 85-102.

Duobiene, J. (2013). Corporate entrepreneurship in organizational lifecycle. Economics and Management, 18(3), 584-595.

Dyduch, W. (2008). Corporate entrepreneurship measurement for improving organizational performance. Journal of Economics and Management, Vol. 4, 15-40.

Engelen, A., Vishal, G., Strenger, L. \& Brettel, M. (2012). Entrepreneurial orientation, firm performance, and the moderating role of transformational leadership behaviors. Journal of Management, 41(4), 1069-1097.

Entebang, H. \& Harrison, R.T. (2012). An integrated definition of corporate entrepreneurship. International Journal of ASEAN Entrepreneurship and Business Development, 1(1), 119-130.

Ferreira, J. (2005). Corporate entrepreneurship: A strategic and structural perspective. New England Journal of Entrepreneurship, 4(2), 59-70.

Garvin, D.A. \& Levesque, L.C. (2006). Meeting the challenge of corporate entrepreneurship. Harvard Business Review, October Issue.

Gautam, V. \& Verma, V. (1997). Corporate entrepreneurship: Changing perspectives. The Journal of Entrepreneurship, 6(2), 233-244.

Guth, W. \& Ginsberg, A. (1990). Corporate Entrepreneurship (Guest Editors' Introduction). University of Illinois at Urbana-Champaign's Academy for Entrepreneurial Leadership Historical Research Reference in Entrepreneurship. Available at SSRN: https://ssrn.com/abstract=1505909.

Hayton, J.C. \& Kelley, D.J. (2006). A competency-based framework for promoting corporate entrepreneurship. Human Resource Management, 45(3), 407-427.

Heavey, C. \& Simsek, Z. (2013). Top management compositional effects on corporate entrepreneurship: The moderating role of perceived technological uncertainty. Journal of Product Innovation Management, 30(5), 837-855.

Heidemann Lassen, A., Gertsen, F. \& Riis, J.O. (2006). The nexus of corporate entrepreneurship and radical innovation. Creativity and Innovation Management, 15(4), 359-372.

Hitt, M.A., Ireland, R.D., Camp, S.M. \& Sexton, D.L. (2001). Strategic entrepreneurship: Entrepreneurial strategies for wealth creation. Strategic Management Journal, 22, 479-491. 
Hornsby, J.S., Naffziger, D., Kuratko, D.F. \& Montagno, R.V. (1993). An interactive model of the corporate entrepreneurship process. Entrepreneurship Theory and Practice, 17(2), 29-37.

Hornsby, J.S., Kuratko, D.F. \& Zahra, S.A. (2002). Middle managers' perception of the internal environment for corporate entrepreneurship: Assessing a measurement scale. Journal of Business Venturing, 17(3), 253-273.

Hostager, T.J., Neil, T.C., Decker, R.L. \& Lorentz, R.D. (1998). Seeing environmental opportunities: Effects of intrapreneurial ability, efficacy, motivation and desirability. Journal of Organizational Change Management, 11(1), 11-25.

Höglund, L. (2009). Affinities in Corporate Entrepreneurship: A Literature Review, Conference Paper.

Ireland, R.D., Covin, G.J. \& Kuratko, D.F. (2009). Conceptualizing corporate entrepreneurship strategy. Entrepreneurship Theory and Practice, 33(1), 19-46.

Jennings, D.F. \& Lumpkin, J.R. (1989). Functioning modeling corporate entrepreneurship: An empirical integrative analysis. Journal of Management, 15(3), 485-502.

Jones, G.R. \& Butler, J.E. (1992). Managing internal corporate entrepreneurship: An agency theory perspective. Journal of Management, 18, 733-749.

Kakapour, S., Morgan, T., Parsinejad, S. \& Wieland, A. (2016). Antecedents of corporate entrepreneurship in Iran: The role of strategic orientation and opportunity recognition. Journal of Small Business \& Entrepreneurship, 28(3), 251-266.

Kearney, C. \& Meynhardt, T. (2016). Directing corporate entrepreneurship strategy in the public sector to public value: Antecedents, components, and outcomes. International Public Management Journal, 19(4), 543-572.

Kellermanns, F.W. \& Eddleston, K. (2006). Feuding families: The management of conflict in family firms. In: P. Poutziouris, K. Smyrnios \& B. Klein (eds.). Family Business Research Handbook (pp. 358-368). Northampton, Ma: Edward Elgar Publishing,

Kelley, D.J., Peters, L. \& O’Connor, G.C. (2009). Intra-organizational networking for innovation-based corporate entrepreneurship, Journal of Business Venturing, 24(3), 221-235.

Knight, G.A. (1997). Cross-cultural reliability and validity of a scale to measure firm entrepreneurial orientation. Journal of Business Venturing, 12(3), 213-225.

Kreiser, P., Kuratko, D.F., Covin, J.G. \& House, M. (2011). Operationalizing Corporate Entrepreneurship Strategy (CES): A Configurational Approach. A paper presented at the annual Academy of Management Conference, San Antonio.

Kuratko, D.F., Ireland, R.D. \& Hornsby, J.S. (2004). Corporate entrepreneurship behavior among managers: A review of theory, research and practice. 
In: A. Katz \& A. Shepherd (eds.). Corporate Entrepreneurship, Advances in Entrepreneurship, Firm Emergence and Growth, Elsevier, Volume 7.

Kuratko, D.F., Montagno, R.V. \& Hornsby, J.S. (1990). Developing an intrapreneurial assessment instrument for an effective corporate entrepreneurial environment. Strategic Management Journal, 11, Special Issue, 49-58.

Kuratko, D.F. \& Audretsch, D. (2009). Strategic entrepreneurship: Exploring different perspectives of an emerging concept. Entrepreneurship Theory and Practice, 33, 1-17.

Lassen, A.H. (2007). Corporate-entrepreneurship: An empirical study of the importance of strategic considerations in the creation of radical innovation. Managing Global Transitions, 5(2), 109-131.

Li, L., Tse, E.C.-Y. \& Zhao, J.-L. (2009). An empirical study of corporate entrepreneurship in hospitality companies. International Journal of Hospitality \& Tourism Administration, 10(3), 213-231.

Ling, Y., Simsek, Z., Lubatkin, M.H. \& Veiga, J.F. (2008). Transformational leadership's role inpromoting corporate entrepreneurship: Examining the CEOTMT interface. Academy of Management Journal, 51(3), 557-576.

Lumpkin, G,T. \& Dess, G.G. (1996). Clarifying the entrepreneurial orientation construct and linking it to performance. Academy of Management Review, 21(1), 135-172.

Macrae, N. (1976). The coming entrepreneurial revolution: A survey. The Economist, 26 (December 25), 41-61.

Macrae, N. (1982). Intrapreneurial Now. The Economist 283 (April 17), 67-72.

Maes, J. (2003). The search for corporate entrepreneurship: A clarification of the concept and its measures. Working Paper Steunpunt OOI, Sept., 1-39.

Maes, J., Sels, L. \& Roodhooft, F. (2005). Modelling the link between management practices and financial performance. Small Business Economics, 25(1), $17-34$.

McFadzean, E., O’Loughlin, A. \& Shaw, E. (2005). Corporate entrepreneurship and innovation. Part 1: The missing link. European Journal of Innovation Management, 8(3), 350-372.

Michalski, T. (2005). Radical innovation through corporate entrepreneurship from a competence-based strategic management perspective. International Journal of Management Practice, 2(1).

Miller, D. \& Friesen, P.H. (1992). Innovation in conservative and entrepreneurial firms: Two models of strategic momentum. Strategic Management Journal, 3(1), 1-25.

Morris, M.H. \& Kuratko, D.F. (2002). Corporate Entrepreneurship. Mason, Ohio: Thompson South-Western. 
Morris, M.H., Kuratko, D.F. \& Covin, J.G. (2008). Corporate Entrepreneurship and Innovation ( $2^{\text {nd }}$ ed.). Mason, Ohio: Thomson South-Western.

Morris, M.H. \& Sexton, D.L. (1996). The Concept of Entrepreneurial Intensity, Implications for Company Performance, Journal of Business Research, $36(1), 5-13$.

Naldi, L., Achtenhagen, L. \& Davidsson, P. (2015). International corporate entrepreneurship among SMEs: A test of Stevenson's Notion of entrepreneurial management. Journal of Small Business Management, 53(3), 780-800.

Narayanan, V., Yang, Y. \& Zahra, S. (2009). Corporate venturing and value creation: A review and proposed framework. Research Policy, 38, 58-76.

Nason, R.S., McKelvie, A. \& Lumpkin, G.T. (2015). The role of organizational size in the heterogeneous nature of corporate entrepreneurship. Small Business Economics, 45, 279-304.

Obłój, T, Obłój, K. \& Pratt, M.G. (2010). Dominant logic and entrepreneurial firms' performance in a transition economy. Entrepreneurship Theory and Practice, 34(1), 151-170.

Paunović, B. (2012). The role of corporate entrepreneurship in solving the competitiveness crisis of large companies. Serbian Association of Economists Journal, 7-8, 343-354.

Phan, P.H., Wright, M., Ucbasaran, D. \& Tan, W.-L. (2009). Corporate entrepreneurship: Current research and future directions. Journal of Business Venturing, 24, 197-205.

Pinchot, G. (1985). Intrapreneuring: Why You Don't Have to Leave the Corporation to Become an Entrepreneur. New York: Harper and Row.

Rauch, A., Wiklund, J. \& Lumpkin, G.T. (2009). Entrepreneurial orientation and business performance: An assessment of past research and suggestions for the future. Entrepreneurship Theory and Practice, 33(3), 761-787.

Romero-Martinez, A.M., Fernández-Rodríguez, Z. \& Vázquez-Inchausti, E. (2010). Exploring corporate entrepreneurship in privatized firms. Journal of World Business, 45(1), 2-8.

Rusak, H. (2016). Corporate entrepreneurship in the arts in Western Australia. The Journal of Arts Management, Law, and Society, 46(4), 153-163.

Sakhdari, K. (2016). Corporate entrepreneurship: A review and future research agenda. Technology Innovation Management Review, 6(8), 5-18.

Sambrook, S. \& Robert, C. (2005). Corporate entrepreneurship and organizational learning: a review of the literature and the development of a conceptual framework. Strategic Change. Briefings in Entrepreneurial Finance, 14(3), 141-155. 
Scheepers, M.J., Hough, J. \& Bloom, J.Z. (2007). Entrepreneurial intensity: A comparative analysis of established companies in South Africa. SAJEMS, $10(2), 238-255$

Schindehutte, M., Morris, M.H. \& Kuratko, D.F. (2000). Triggering events, corporate entrepreneurship and the marketing function. Journal of Marketing Theory and Practice, 8(2), 18-30.

Schollhammer, H. (1982). Internal corporate entrepreneurship. In: C.A. Kent, D.L. Sexton \& K.H. Vesper (eds.). Encyclopedia of Entrepreneurship, Englewood Cliffs, NJ: Prentice-Hall.

Schumpeter, J.A. (1912/1934). Theory of Economic Development. Cambridge, MA: Harvard University Press.

Schumpeter, J. (1942). Capitalism, socialism and democracy. New York, NY: Harper \& Row.

Sharma, P. \& Chrisman, J.J. (1999). Toward a reconciliation of the definitional issues in the field of corporate entrepreneurship. Entrepreneurship Theory and Practice, 23(3), 11-27.

Simsek, Z. \& Heavey, C. (2011). The mediating role of knowledge-based capital for corporate entrepreneurship effects on performance: A study of small- to medium-sized firms. Strategic Entrepreneurship Journal, 5(1), 81-100.

Simsek, Z., Heavey, C., Veiga, J.F. \& Souder, D. (2009). A typology for aligning organizational ambidexterity's conceptualizations, antecedents, and outcomes. Journal of Management Studies, 46(5), 864-894.

Stevenson, H.H. \& Jarillo, J.C. (1990). A paradigm of entrepreneurship: Entrepreneurial management. Strategic Management Journal, 11 (special issue), $17-27$.

Stopford, J.M. \& Baden-Fuller, C. (1994). Creating corporate entrepreneurship. Strategic Management Journal, 15(7), 521-536.

Tajeddini, K \& Mueller, S.L. (2012). Corporate entrepreneurship in Switzerland: Evidence from a case study of Swiss watch manufacturers. International Entrepreneurship Management Journal, 8, 355-372.

Thornberry, N.E. (2003). Corporate entrepreneurship: Teaching managers to be entrepreneurs. Journal of Management Development, 22(4), 329-344.

Ucbasaran, D., Westhead, P. \& Wright, M. (2001). The focus of entrepreneurial research: Contextual and process issues. Entrepreneurship: Theory \& Practice, 25(4), 57-80.

Vesper, K.H. (1984). Three Faces of Corporate Entrepreneurship: A Pilot Study. Seattle: University of Washington, Graduate School of Business. 
Von Hippel, E. (1977). Transferring process equipment innovations from user-innovators to equipment manufacturing firms. $R \& D$ Management, 8(1), 13-22.

West, G.P. (2007). Collective cognition: When entrepreneurial teams, not individuals, make decisions. Entrepreneurship: Theory and Practice, 31(1), 77-102.

Wolcott, R.C. \& Lippitz, M.J. (2007). The four models of corporate entrepreneurship. MIT Sloan Management Review, 49(1), 75-82.

Wójcik-Karpacz, A. (2016). The choice of measures for entrepreneurial orientation: Dilemmas and possible solutions. Research Papers of Wrocław University of Economics, No. 444, 594-608.

Wu, C.-H., Parker, S.K. \& de Jong, J.P.J. (2011). Need for cognition as an antecedent of individual innovation behavior. Journal of Management, 40(6), 1511-1534.

Yildiz, M.L. (2014). The effects of organizational culture on corporate entrepreneurship. International Journal of Business and Social Science, 5(1), 35-44.

Yiu, D.W. \& Lau, C.-M. (2008). Corporate entrepreneurship as resource capital configuration in emerging market firms. Entrepreneurship: Theory and Practice, 32(1), 37-57.

Zahra, S. (1991). Predictors and financial outcomes of corporate entrepreneurship: An exploratory study. Journal of Business Venturing, 6(4), 259-286.

Zahra, S.A. (1993). Environment, corporate entrepreneurship, and financial performance: A taxonomic approach. Journal of Business Venturing, 8(4), 319-340.

Zahra, S.A. (1996). Governance, ownership and corporate entrepreneurship: The moderating impact of industry technological opportunities. Academy of Management Journal, 39(6), 1713-1735.

Zahra, S.A., Neubaum, D.O. \& Huse, M. (2000). Entrepreneurship in medium-size companies: Exploring the effects of ownership and governance systems. Journal of Management, 26(5), 947-976.

Zajac, E.J., Golden, B.R. \& Shortell, S.M. (1991). New organizational forms for enhancing innovation: The case of internal corporate ventures. Management Science, 37(2), 170-184.

Zimmerman, B.J. (1990). Self-regulated learning and academic achievement: An overview. Educational Psychologist, 25(1), 3-17.

Żur, A. (2013). Entrepreneurial orientation and firm performance: Challenges for research and practice. Entrepreneurial Business and Economics Review, $1(2), 7-28$. 


\title{
PRZEDSIĘBIORCZOŚĆ KORPORACYJNA. PRZEGLĄD LITERATURY I PERSPEKTYWY PRZYSZKYCH BADAŃ
}

\begin{abstract}
Abstrakt
Tło. Przedsiębiorczość korporacyjna postrzegana jest jako podejście niezbędne do pobudzania innowacyjności i kreatywności w organizacjach $\mathrm{w}$ celu zwiększenia szans rynkowych. Niniejszy artykuł analizuje tę koncepcję, cieszącą się dość dużym zainteresowaniem $\mathrm{w}$ ciagu ostatnich trzydziestu lat w krajach anglosaskich. Podobnie jak w przypadku wielu innych zjawisk, także w odniesieniu do przedsiębiorczości korporacyjnej, dyskusja ta dostarczyła licznych konceptualizacji i z tego względu nie ma jednej uniwersalnej definicji tej koncepcji. W tym kontekście, poszukiwanie odpowiedniej podstawy do zrozumienia i opisania zjawiska przedsiębiorczości korporacyjnej stawia środowisku badaczy przedsiębiorczości sporo wyzwań.
\end{abstract}

Cele badawcze. Niniejszy artykuł ma na celu stworzenie takiej podstawy poprzez doprecyzowanie pojęcia przedsiębiorczości korporacyjnej, dzięki zrealizowanemu przeglądowi literatury oraz poprzez identyfikację istniejących luk badawczych.

Metodologia. Stosowana metodologia jest systematyczny przegląd literatury. Bazy danych EBSCO oraz Taylor \& Francis posłużyły jako źródło zgromadzonej w ramach badań literatury.

Kluczowe wnioski. Analiza potwierdza potrzebę bardziej jakościowych i rygorystycznych analiz w tej dziedzinie oraz zawiera szereg zaleceń dla przyszłych badań. Główne konkluzje zachęcają do różnicowania badań pod kątem sektorów, a największa zidentyfikowana luka występuje w sektorze usług. Istnieje również potrzeba bardziej ustrukturyzowanej klasyfikacji miar zjawiska przedsiębiorczości korporacyjnej w zależności od faktycznego celu badań: jego poprzedników lub efektów.

Słowa kluczowe: przedsiębiorczość korporacyjna, intraprzedsiębiorczość, przedsięwzięcie korporacyjne. 